\title{
Findings of Fat Containing Hepatocellular Carcinoma on Contrast-enhanced Ultrasound with Sonazoid: A Case Report
}

\author{
Mengna He, MD, PHD, Lu Zhu, MD, Tianan Jiang, MD, PHD \\ Department of Ultrasound, the First Affiliated Hospital, College of Medicine, Zhejiang University, Zhejiang Province, China \\ Received January 09, 2020; revision received February 24, 2020; accepted March 26, 2020
}

Abstract: Contrast-enhanced ultrasound (CEUS) has become more recognized in the diagnosis of hepatocellular carcinoma (HCC). Fat containing HCC often presents as hyper-echoic in gray scale ultrasound and confuses the diagnosis. Here we showed a case of fat containing HCC which was diagnosed by CEUS with Sonazoid, and its histopathologic features, immunohistochemical profile, surgical treatment, patient's follow up information were also presented.

Key words: Hepatocellular carcinoma; Sonazoid; Contrast agent; Fat-containing

Advanced Ultrasound in Diagnosis and Therapy 2021;02:098-101

DOI: 10.37015/AUDT.2021.200003

$\mathrm{T}$ he high incidence of HCC and its threat to health is a global burden. Identification of HCC in an early stage is extremely important since this kind of patient can receive curative treatment before the tumor spreading; and then the quality of life can be improved and the economic cost of society can be reduced [1]. With the development of imaging techniques such as contrast enhanced ultrasound (CEUS) [2], most HCC can be correctly diagnosed before operation, due to their typical enhancement pattern: hyper-enhancement in the arterial phase followed by mile-late wash-out in the portal phase and delayed phase, which is different from most benign lesions.

However, some well-differentiated HCC show isoenhancement at delayed phase and have been reported to have various enhancement patterns [3], which depends on the degree of carcinogenesis including the increase of arterial flow and decrease of portal supplying [4]. And if they contain fat, diagnosis can be even more challenging since fat containing HCC often presents as hyperechoic on gray scale ultrasound, which is different from common HCC [5], and the CEUS manifestation of this kind of HCC is always atypical. The reason might be that fat containing was usually found in the welldifferentiated HCC, and with the increased histologic grade, fat containing became infrequent [6].

Sonazoid (Daiichi Sankyo, Tokyo, Japan) is a novel ultrasound contrast agent and it was officially launched in China in March 2019. It provides hepatic parenchymaspecific contrast images because of its accumulation in the Kupffer cells of the liver [7]. Whether SonazoidCEUS can provide meaningful diagnostic information for fat containing HCC needs to be investigated.

This report is the first time to describe the SonazoidCEUS features of fat-containing HCC. The low staining of the lesion in the Kupffer phase provided valid information and increased the confidence in the diagnosis of HCC.

\section{Case Report}

\section{Chief complaints}

A 36-year-old male complained of liver cirrhosis and focal liver mass for 6 months during regular gray scale ultrasound follow-up, without any obvious positive symptoms and signs when he presented at our

\footnotetext{
* Corresponding author: Department of Ultrasound, the First Affiliated Hospital, College of Medicine, Zhejiang University, Hangzhou 310003, Zhejiang Province, China.

e-mail: tiananjiang@zju.edu.cn unrestricted use, distribution and reproduction in any medium provided that the original work is properly attributed.
} 
hospital. Local ultrasound report showed liver cirrhosis, splenomegaly and liver mass suspected as HCC.

\section{History of present illness}

The patient was diagnosed with hepatitis $B$ virus (HBV) infection for 20 years, and two years ago the patient visited to our hospital for routine follow-up, laboratory examinations showed that the value of HBVDN was $3.82 \times 10^{7} \mathrm{IU} / \mathrm{mL}$, the content of hepatitis B surface antigen (HBsAg) was $4520.35 \mathrm{IU} / \mathrm{mL}$, and the liver function index was normal with $40 \mathrm{U} / \mathrm{L}$ Alanine aminotransferase (ALT) and $35 \mathrm{U} / \mathrm{L}$ Aspartate transaminase (AST); liver ultrasound examination showed no liver cirrhosis or focal liver lesions (FLLs), and the patient began to take Entecavir capsules 0.5 mg, Qd regularly. Six months ago, the follow-up examinations showed that the value of ALT and AST was $72 \mathrm{U} / \mathrm{L}$ and $68 \mathrm{U} / \mathrm{L}$ respectively, HBV-DN was $2.55 \times 10^{7} \mathrm{IU} / \mathrm{mL}$, HBsAg was $1973.27 \mathrm{IU} / \mathrm{mL}$. The liver ultrasound showed liver cirrhosis, splenomegaly, multiple regenerating nodules ( $\mathrm{RNs}$ ) with a maximum diameter of $1.3 \mathrm{~cm}$ and the patient continued to be followed up regularly. The latest examination was two days ago and the results showed that the value of ALT and AST was $55 \mathrm{U} / \mathrm{L}$ and $51 \mathrm{U} / \mathrm{L}$ respectively, HBV-DN was $1.89 \times 10^{4}$ $\mathrm{IU} / \mathrm{mL}$, HBsAg was $1786.05 \mathrm{IU} / \mathrm{mL}$. The value of Alpha fetoprotein (AFP) was $208.1 \mathrm{ng} / \mathrm{mL}$, the liver ultrasound showed liver cirrhosis, splenomegaly, multiple FLLs and the largest lesion with a maximum diameter of $3.0 \mathrm{~cm}$ which was suspected as HCC (Fig. 1).
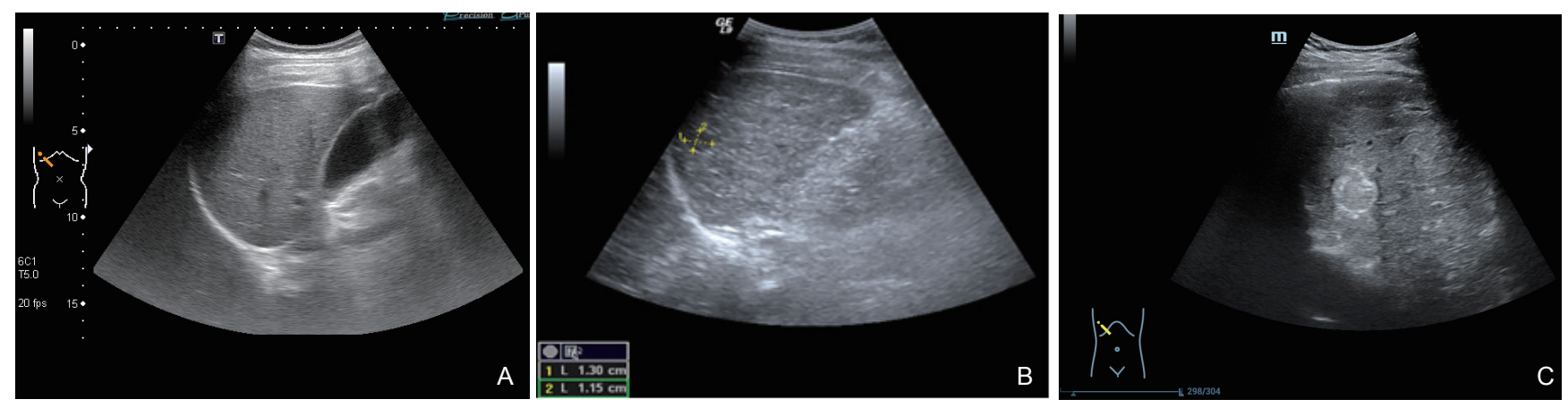

Figure 1 Gray scale ultrasound images of the patient's liver 2 years ago (A), 6 months ago (B); 2 days ago (C).

\section{Family history}

The patient's mother had HBV related liver cirrhosis and three sisters had chronic hepatitis $\mathrm{B}$, but none of them had a history of HCC.

\section{Physical examination}

The patient's basic information showed no skin or scleral yellow staining, but several spider nevus can be seen in the anterior part of the neck and double forearms.

\section{Laboratory examination}

Blood analysis showed white blood cells at $3.4 \times 10^{9} / \mathrm{L}$, with light decrease of neutrophilic granulocyte percentage at $40.1 \%$ and increase of lymphocyte percentage at $47.8 \%$. The value of ALT and AST was $41 \mathrm{U} / \mathrm{L}$ and $39 \mathrm{U} / \mathrm{L}$ respectively, HBV-DN was $2.67 \times 10^{4} \mathrm{IU} / \mathrm{mL}, \mathrm{HBsAg}$ was $1796.77 / \mathrm{mL}$. The partial thromboplastin, prothrombin times and D-dimers were normal. The serum hepatic fibrosis index increased including Urine collagen IV at $167.92 \mathrm{ng} / \mathrm{mL}$, Urine Laminin at $117.14 \mathrm{ng} / \mathrm{mL}$, and serum hyaluronic acid at $270.77 \mathrm{ng} / \mathrm{mL}$ and type III Procollagen n-terminal peptide at $15.08 \mathrm{ng} / \mathrm{mL}$. The value of tumor abnormal protein (TAP) and AFP were $127 \mathrm{MAU} / \mathrm{mL}$ and $235 \mathrm{ng} / \mathrm{mL}$ respectively.

\section{Imaging examination}

All ultrasound examinations were performed with a MyLab Classic system (Easote, Italy) equipped with a convex probe (3.5 MHz). Gray scale ultrasound showed a hyper-echoic lesion with clear margin and round shape in the segment 7, Doppler Flow Imaging (CDFI) showed no obvious flow signal in the lesion. The mechanical index (MI) was set at 0.20 for the Sonazoid CEUS examination. Sonazoid was used with a recommended dose of 0.015 $\mathrm{mL} / \mathrm{kg}$ and injected into the cubital vein. SonazoidCEUS showed homogenous hyper-enhancement of the lesion in arterial phase and prolonged iso-enhanced with the peripheral parenchyma in the portal and delayed vascular phase. Post-vascular phase observation showed a defect of perfluorobutane contrast agent of the lesion in Kupffer phase (Fig. 2), and HCC was suspected.

\section{Final diagnosis and treatment}

Considering the low uptake of Sonazoid in Kupffer phase by the lesion and the fact that the patient had 20 years' HBV infection history and liver cirrhosis, the patient received a liver biopsy (LB) for the lesion and after LB, radiofrequency ablation (RFA) was performed. 


\section{Outcome and follow-up}

The pathological reports showed the lesion was a well-differentiated HCC and immunochemistry staining confirmed it including the presence of hepatocyte and GPC-3 and absence of CK7, CK19 and CD68 (Fig.
3). The patient was discharged two days after RFA and one month later, the patient came to our hospital for reexamination with CE-MRI and results showed complete remission of the lesion.
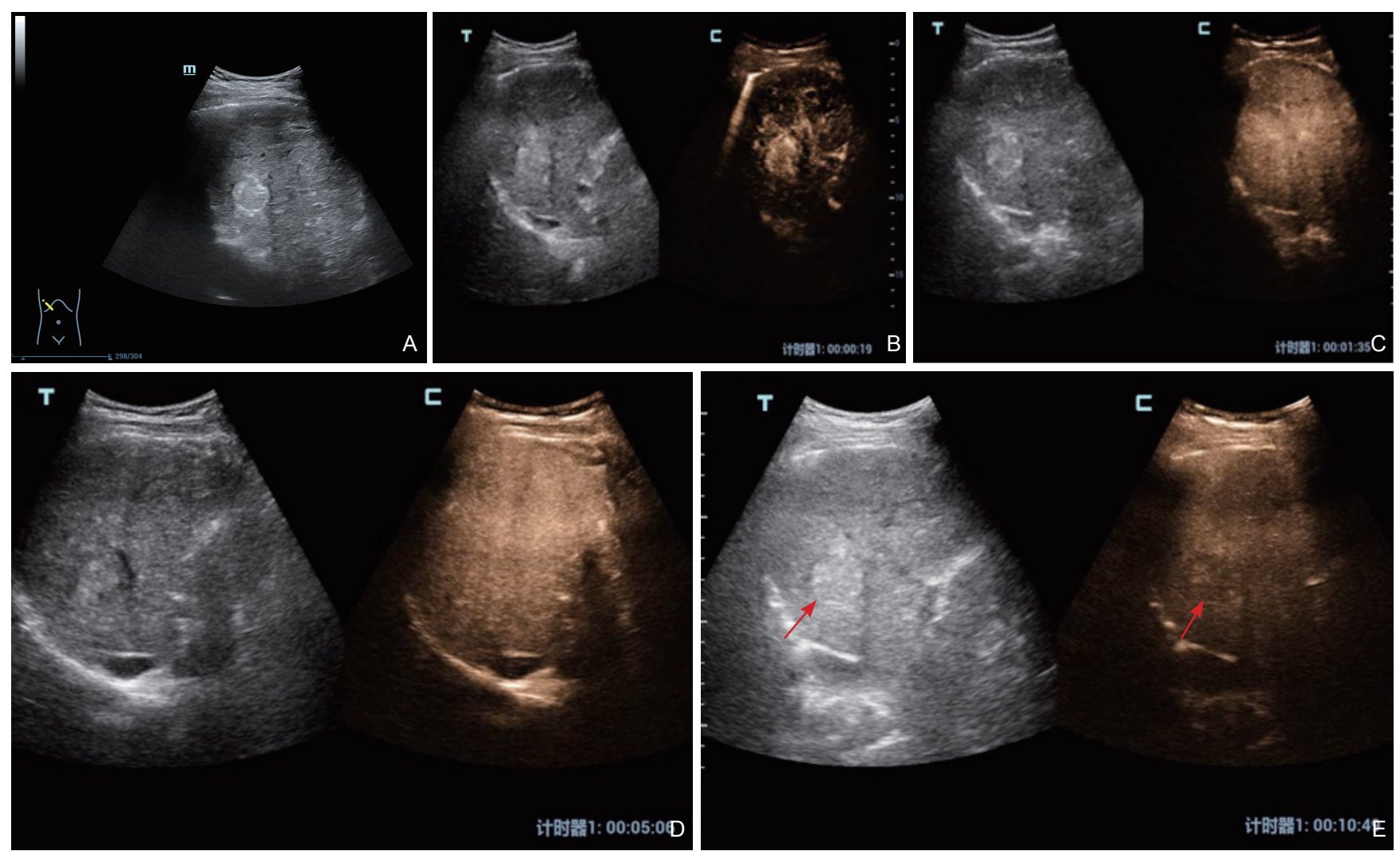

Figure 2 The images of Sonazoid-CEUS. (A) Gray scale ultrasound (left) showed a hyper-echoic, clear margin, round shape lesion in Segment VII; (BD) the lesion was hyper-enhanced in the arterial phase with consistent iso-enhanced in the portal and late phase and cannot be distinguished; (E) it showed a defect of contrast agent in Kupffer phase (arrow).

\section{Discussion}

In the background of liver cirrhosis, a lesion containing fat was special but easily overlooked for HCC, and the reason for fat developed in HCC is still unknown. Most researches supported the relative ischemia theory during the process of reconstruction of blood supply ratio between hepatic artery and portal vein, so for the advanced HCC lesions especially large ones with established arterial system, containing fat was rare [5]. And due to the establishment of unpaired arteries, the contrast enhanced features of advanced HCC lesions are hyper-enhancement in the arterial phase and quickly wash out in the portal phase.

However, what often confused us was well-differentiated HCC which might show sustained enhancement during the portal phase and delayed phase, and if this type of HCC also contains fat, then it will become increasingly difficult to be correctly diagnosed. Because some benign focal liver lesions (FLLs) like hepatic Angiomyolipoma
(HAML), focal nodular hyperplasia (FNH) can also contain fat. Besides, they sometimes shared similar imaging characteristics with the case in this study: hyperenhanced in arterial phase and consistent hyper/isoenhanced in the portal phase and delayed phase.

Fortunately, with the application of Sonazoid, several studies had confirmed the iso-uptake of Sonazoid contrast agent at the Kupffer phase of HAML and FNH, compared with peripheral liver parenchyma $[8,9]$. Meanwhile, there was pathological evidence supporting that CD68 staining positive cells were found [9], which means the existence of Kupffer cells or migrating macrophages in these two kinds of FLLs. And the application of Sonazoid, CD68 positive cell-specific ultrasound contrast agent, enables the preoperative imaging diagnosis of FLLs to be accurately to the cellular level. And the low Sonazoiduptake in Kupffer phase could be a promising feature for differentiating HCC from some benign kinds of FLLs. 

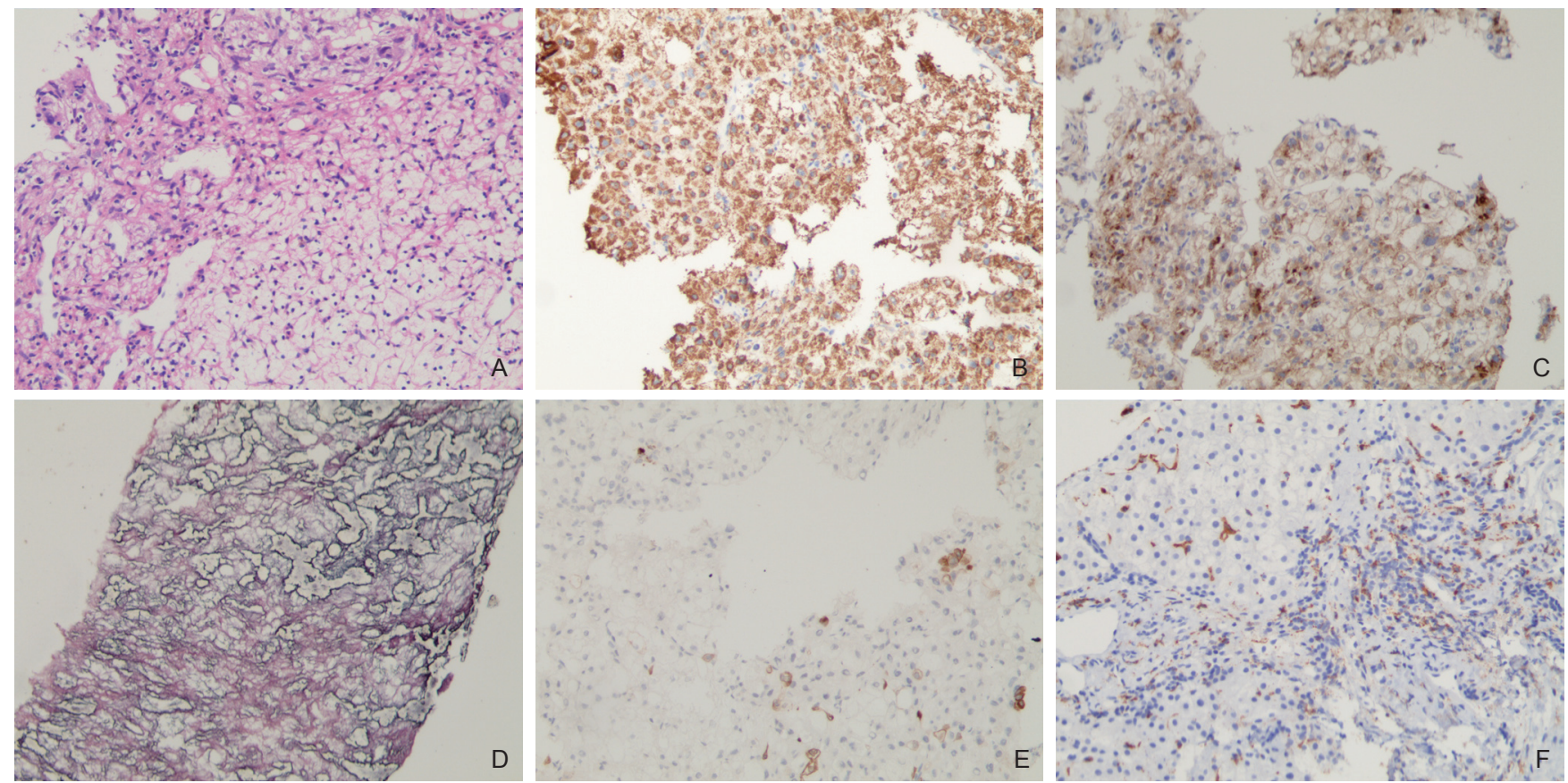

Figure 3 The biopsy pathologic image of the lesion, hematoxylin-eosin stain (A). (B-F) Immunohistochemical images of the lesion: Hepatocellular (+), GPC-3 (+), Reticular fiber staining (-), CK7 (-) and CD68 (-) from B to F, respectively.

\section{Conclusion}

We have presented a case of fat containing $\mathrm{HCC}$ and described the Sonazoid-CEUS features here, which might be helpful in the diagnosis of a hyper-echoic fat containing HCC.

\section{Funding}

The article was funded by the Zhejiang provincial natural science fund (LQ20H180013).

\section{Conflict of Interest}

All authors concur with the submission and have no financial \& commercial conflicts of interest related to this work.

\section{References:}

[1] Ronot M, Purcell Y, Vilgrain V. Hepatocellular carcinoma: current imaging modalities for diagnosis and prognosis. Dig Dis Sci 2019; 64:934-950..

[2] European Association For The Study Of The Liver; European Organisation For Research And Treatment Of Cancer. EASLEORTC clinical practice guidelines: management of hepatocellular carcinoma. J Hepatol 2012;56:908-43.
[3] Suzuki K, Okuda Y, Ota M, Kojima F, Horimoto M. Diagnosis of hepatocellular carcinoma nodules in patients with chronic liver disease using contrast-enhanced sonography: usefulness of the combination of arterial- and kupffer-phase enhancement patterns. $J$ Ultrasound Med 2015;34:423-33.

[4] Choi JY, Lee JM, Sirlin CB. CT and MR imaging diagnosis and staging of hepatocellular carcinoma: part II. Extracellular agents, hepatobiliary agents, and ancillary imaging features. Radiology 2014;273:30-50.

[5] Tekath M, Klotz T, Montoriol PF, Joubert-Zakeyh J, Garcier JM, Da Ines D. Fat-containing lesions of the liver: a pictorial essay. Diagn Interv Imaging 2015; 96:201-11.

[6] Kutami R, Nakashima Y, Nakashima O, Shiota K, Kojiro M. Pathomorphologic study on the mechanism of fatty change in small hepatocellular carcinoma of humans. J Hepatol 2000; 33:282-9.

[7] Yang WY, Park HS, Kim YJ, Yu MH, Jung SI, Jeon HJ. Visibility of focal liver lesions: Comparison between kupffer phase of CEUS with sonazoid and hepatobiliary phase of gadoxetic acid-enhanced MRI. $J$ Clin Ultrasound 2017; 45:542-550.

[8] Lee J, Jeong WK, Lim HK, Kim AY. Focal Nodular Hyperplasia of the Liver: Contrast-Enhanced Ultrasonographic Features With Sonazoid. J Ultrasound Med 2018;37:1473-1480.

[9] Tochio H, Tamaki E, Imai Y, Iwasaki N, Minowa K, Chung H, Suginoshita Y, Inokuma T, Kudo M. CD68-Positive Cells in Hepatic Angiomyolipoma. Oncology 2017;92:35-39. 